\title{
To study the feto-maternal outcome in cases with previous surgical intervention for first trimester abortion on subsequent pregnancy and to compare with cases without history of previous abortion
}

\author{
Kritika Tanwar*, Bani Sarkar, Anjali Chauhan
}

Department of Obstetrics and Gynaecology, Dr RML Hospital, New Delhi, India

Received: 05 August 2020

Accepted: 09 September 2020

\author{
*Correspondence: \\ Dr. Kritika Tanwar, \\ E-mail: drkritikatanwar@gmail.com
}

Copyright: () the author(s), publisher and licensee Medip Academy. This is an open-access article distributed under the terms of the Creative Commons Attribution Non-Commercial License, which permits unrestricted non-commercial use, distribution, and reproduction in any medium, provided the original work is properly cited.

\begin{abstract}
Background: To study the Feto-maternal outcome in cases with previous surgical intervention for first trimester abortion on subsequent pregnancy and to compare with cases without history of previous abortion.

Methods: A cross sectional observational study was conducted over a period of two years. Study included 80 consenting females at 28 weeks POG. Females with history of prior surgical abortion just before the present pregnancy were included as cases $(n=40)$ and rest as controls $(n=40)$. Detailed obstetric history was taken, subjects were then followed till delivery and feto-maternal outcomes such as period of gestation, mode of delivery birth weight, Apgar score, increased NICU stay at the time of delivery were recorded and analyzed.

Results: We observed that the women with history of induced surgical abortion were at increased risk of Pre term birth $(52.5 \%$, p value0.006), Cesarean section (40\%, p value 0.012), Low birth weight (47.5\%, p value <0.001), increased NICU stay $(32.5 \%$, p value 0.027$)$ compared to primi-gravida controls.

Conclusions: We concluded that women with previous history of induced surgical abortions were at increased risk of preterm birth, very preterm birth and low birth weight babies, NICU stay in the subsequent pregnancies. The risk of caesarean was found to be increased in women with previous induced abortions exposing the women to the morbidity associated with the $\mathrm{C}$-section. Hence patient with prior history of surgical first trimester abortion needs more vigilant monitoring during antenatal period in subsequent pregnancy.
\end{abstract}

Keywords: Abortion, APGAR score, Feto-maternal

\section{INTRODUCTION}

Induced abortion is the most common gynecological procedure performed in women. The number of abortions has been increasing every year, and hence the need to combat its complication is important. Induced abortion is the main concern for females in early reproductive period, and who may want child in future. There are many cases of abortion which are either spontaneously, medically or surgically terminated by vacuum aspiration, Dilatation and Evacuation or Dilatation and Curettage. Even though, there is a decline in the number of unintended pregnancies, but the absolute number of such pregnancies is relatively high in the developing countries. It is estimated that nearly $40 \%$ of the pregnancies in developing countries are unintended, either not wanted at all or mistimed. ${ }^{1}$ In a developing nation like India, this constitutes a huge number. Hence it is important to evaluate the subsequent adverse obstetric outcomes of prior induced surgical abortion in order to reduce the morbidity associated with it.

Data from registered medical termination of pregnancies (MTP) centers (India) revealed that 667,294 women 
underwent medical or surgical abortion in $2013-2014$ and 701,421 abortions were reported in 2014-2015, thus showing a rising trend. ${ }^{2}$ But the effect of surgical abortion on subsequent pregnancy has been studied in very few centers in India and thus limited data is available on long term complications of induced abortion such as pre-term labor, low birth weight baby, small for gestation age etc.

Number of long term adverse outcome are associated with previous surgical termination of pregnancy which includes pre term birth, low birth weight baby, small for gestation age infants, placental complications. ${ }^{3}$ Some studies have also shown induced abortion is associated with an increased risk of first trimester miscarriage and lead to complications in later part of subsequent pregnancy. ${ }^{4}$ The effect of surgical abortion on subsequent pregnancy has been studied in very few centers in India and thus limited data is available on long term complications of induced abortion such as pre-term labor, low birth weight baby, small for gestation age.

The present study was done to see the long term effect of surgical abortion in subsequent pregnancy by documenting multiple fetal and maternal outcomes in selected group of patients and comparing the same with matched controls.

\section{METHODS}

The present study is a Cross sectional observational study, conducted at the Department of Obstetrics and Gynecology, PGIMER, Dr. Ram Manohar Lohia Hospital between 1st November 2017 to 31st march 2019. Study included 80 consenting subjects, fulfilling the inclusion and exclusion criteria, from 28 weeks period of gestation. There were 40 patients included in both cases and control. Amongst the cases 40 females between 18 to 40 years of age, with period of gestation 28 weeks or more with history of one first trimester surgical abortion by dilatation and evacuation, just before this pregnancy were included as cases. Cases included had no antenatal medical complication at time of entering the study.

Amongst control 40 primi-gravida with period of gestation more than equal to 28 weeks with no antenatal medical illness at time of enrollment in the study were included. Both the groups were followed till delivery and fetal and maternal outcomes were measured. Fetal and maternal outcome included in the study were:

\section{Maternal outcomes}

- Period of gestation of delivery

- Mode of delivery

\section{Fetal outcomes which included}

- Weight of the baby
Complications in the baby such as meconium stained liquor, fetal distress, congenital malformations, low Apgar score, neonatal intensive care unit (NICU) stay.

\section{Inclusion criteria}

History of surgical intervention for first trimester abortion for cases and no history of surgical abortion amongst controls. Period of gestation more than 28 weeks. Age between 18-40 years. No antenatal complication at time of enrollment.

\section{Exclusion criteria}

Known case of Incompetent cervix, uterine malformation and uterine fibroid. History of diabetes mellitus, chronic hypertension, renal disease. Multiple gestation. History of trophoblastic disease. Prior low birth weight, pre term labor or recurrent pregnancy loss.

Both the cases and controls were followed till delivery and fetal and maternal outcomes were recorded. Following data was recorded at the time of enrollment:

Detail history including; demographic details, any chief complaints, Menstrual history, detailed obstetrical history, past medical or surgical history, family history and personal history.

\section{Detail examination}

Routine antenatal investigation as per hospital protocol. These patients were followed till delivery and fetomaternal outcomes will be recorded.

\section{Statistical analysis}

Statistical testing was conducted with the statistical package for the social science system version SPSS 17.0. Continuous variables are presented as mean $\pm \mathrm{SD}$, and categorical variables are presented as absolute numbers and percentage. The comparison of normally distributed continuous variables between the groups was performed using Student's t test. Nominal categorical data between the groups were compared using Chi-squared test or Fisher's exact test as appropriate. With regard to the prediction of cases, the Odds ratio $(95 \% \mathrm{CI})$ of each risk factors were calculated. For all statistical tests, a $\mathrm{P}$ value less than 0.05 was taken to indicate a significant difference.

\section{RESULTS}

As per the aim of our study, primary outcomes were assessed using multiple parameters, such as maternal factor which include pre- term labor, mode of delivery and fetal outcomes such as; Small for gestational age, NICU stay, complications in baby, low Birth Weight Baby. 
After assessing the records, factor such as age, BMI and socio economic status were comparable in both the cases and control group. Following maternal and fetal outcomes were obtained.

\section{Maternal outcomes}

It was observed that most of the patient with history of first trimester surgical abortion delivered before 37 weeks. There was significant correlation between history of first trimester surgical abortion and late pre-term birth (32weeks to 37 weeks of period of gestation) (p 0.006, odds ratio $3.89,95 \% \mathrm{CI}$ ).

Table 1: Period of gestation at which patient delivered.

\begin{tabular}{|c|c|c|c|c|c|c|}
\hline \multirow[b]{2}{*}{ POG at delivery } & \multicolumn{2}{|l|}{ Cases } & \multicolumn{2}{|l|}{ Controls } & \multirow[b]{2}{*}{ p value } & \multirow[b]{2}{*}{ Odds ratio $(95 \% \mathrm{CI})$} \\
\hline & Frequency & $\%$ & Frequency & $\%$ & & \\
\hline 28-32 weeks & 1 & $2.5 \%$ & $\mathrm{O}$ & $0 \%$ & 1.000 & $1.67(0.10-28.32)$ \\
\hline 32-37 weeks & 21 & $52.5 \%$ & 9 & $22.5 \%$ & 0.006 & $3.89(1.47-10.31)$ \\
\hline >37 weeks & 18 & $45.0 \%$ & 31 & $77.5 \%$ & 0.006 & - \\
\hline Total & 40 & $100.0 \%$ & 40 & $100.0 \%$ & & \\
\hline
\end{tabular}

There were $21(52.5 \%$ ) late pre term births (32 weeks to 37 weeks) amongst cases whereas only 9 (22.5\%) female delivered at same gestation. One $(2.5 \%)$ female amongst cases had very pre term delivery ( 28 weeks to 30 weeks of gestation) (p 1.000, odds ratio 1.67, 95\% CI).

On the other hand, amongst cases no patient delivered between 28 to 32 weeks period of gestation. 18 (45\%) patient delivered at period of gestation more than 37 weeks amongst the cases whereas in control group 31 (77.5\%) delivered at more than 37 weeks. P value was found to be 0.006 for late pre term birth, which was statistically significant amongst cases than controls. There was statistically significant correlation between cesarean section and history of first trimester surgical intervention, $\mathrm{p}$ value 0.012

Table 2: Mode of delivery amongst cases and controls.

\begin{tabular}{|c|c|c|c|c|c|c|}
\hline \multirow{2}{*}{ Mode of delivery } & \multicolumn{2}{|l|}{ Cases } & \multicolumn{2}{|l|}{ Controls } & \multirow{2}{*}{$\begin{array}{l}\text { p } \\
\text { value }\end{array}$} & \multirow{2}{*}{ Odds ratio (95\% CI) } \\
\hline & Frequency & $\%$ & Frequency & $\%$ & & \\
\hline NVD & 24 & $60.0 \%$ & 34 & $85.0 \%$ & 0.012 & - \\
\hline LSCS & 16 & $40.0 \%$ & 6 & $15.0 \%$ & 0.012 & $3.78(1.29-11.06)$ \\
\hline Instrumental Delivery & 0 & $0.0 \%$ & 0 & $0.0 \%$ & - & - \\
\hline Total & 40 & $100 \%$ & 40 & $100 \%$ & & \\
\hline
\end{tabular}

It was observed in 16 out of $40(40 \%)$ cases were cesarean compared to $6(15 \%)$ in control group. (P; 0.012 , odds ratio $3.78,95 \% \mathrm{CI})$. Amongst cases there were $60 \%$ normal vaginal delivery and $85 \%$ amongst control group. There were no instrumental delivery amongst cases and controls.

\section{Fetal outcomes}

It was seen that the mean birth weight were $2.6 \mathrm{~kg}$ and $2.8 \mathrm{~kg}$ in cases and controls respectively. There was a significant correlation between low birth weights of baby $(1.5-2.5 \mathrm{~kg})$ with $\mathrm{p}$ value 0.001 . There were $19(47.5 \%)$ babies amongst cases who were low birth weight (1.5 to $2.5 \mathrm{~kg}$ ) and only $5(12.5 \%)$ amongst control group. Minimum birth weight seen in cases was $800 \mathrm{gm}$. There was 1 baby under study group whose weight was less than $1 \mathrm{~kg}(2.5 \%)$ and was $800 \mathrm{~g}$. Out of 40 births, 34 $(85 \%)$ babies born to control mothers were weighing more than $2.5 \mathrm{~kg}$ and $47.5 \%$ amongst cases. APGAR score at $1 \mathrm{~min}$ of birth was compared, it was observed 7 (17.5\%) out of 40 babies born had Apgar score less than 7 amongst cases and $3(7.5 \%)$ amongst control.

Table 3: Correlation of birth weight amongst cases and controls.

\begin{tabular}{|c|c|c|c|c|c|}
\hline \multirow{2}{*}{$\begin{array}{l}\text { Baby } \\
\text { weight }\end{array}$} & \multicolumn{2}{|l|}{ Cases } & \multicolumn{2}{|l|}{ Controls } & \multirow{2}{*}{$\begin{array}{l}\mathbf{p} \\
\text { value }\end{array}$} \\
\hline & Frequency & $\%$ & Frequency & $\%$ & \\
\hline$<1 \mathrm{Kg}$ & 1 & $\begin{array}{l}2.5 \\
\%\end{array}$ & 0 & $\begin{array}{l}0.0 \\
\%\end{array}$ & 1.000 \\
\hline $\begin{array}{l}1.0- \\
1.5 \mathrm{Kg}\end{array}$ & 1 & $\begin{array}{l}2.5 \\
\%\end{array}$ & 1 & $\begin{array}{l}2.5 \\
\%\end{array}$ & 1.000 \\
\hline $\begin{array}{l}1.5- \\
2.5 \mathrm{Kg}\end{array}$ & 19 & $\begin{array}{l}47 . \\
5 \%\end{array}$ & 5 & $\begin{array}{l}12.5 \\
\%\end{array}$ & $\begin{array}{l}<0.0 \\
01\end{array}$ \\
\hline $\begin{array}{l}>2.5 \\
\mathrm{Kg}\end{array}$ & 19 & $\begin{array}{l}47 . \\
5 \%\end{array}$ & 34 & $\begin{array}{l}85.0 \\
\%\end{array}$ & $\begin{array}{l}<0.0 \\
01\end{array}$ \\
\hline Total & 40 & $\begin{array}{l}100 \\
\%\end{array}$ & 40 & $\begin{array}{l}100 \\
\%\end{array}$ & \\
\hline
\end{tabular}


There were 40 births in each group, out of which 37 babies amongst cases were alive and healthy, $1(2.5 \%)$ baby died at 29 week of gestation with weight being
$800 \mathrm{gms}$ and one baby was intubated after delivery as baby had respiratory distress due to meconium aspiration.

Table 4: Comparison of neonatal outcomes.

\begin{tabular}{|c|c|c|c|c|c|}
\hline \multirow{2}{*}{ Neonatal outcome } & \multicolumn{2}{|l|}{ Cases } & \multicolumn{2}{|l|}{ Controls } & \multirow{2}{*}{ p value } \\
\hline & Frequency & $\%$ & Frequency & $\%$ & \\
\hline Alive and healthy & 37 & $92.5 \%$ & 39 & $97.5 \%$ & 1.000 \\
\hline Died & 1 & $2.5 \%$ & 0 & $0.0 \%$ & 1.000 \\
\hline MSL & 1 & $2.5 \%$ & 1 & $2.5 \%$ & 1.000 \\
\hline Still Birth & 0 & $0.0 \%$ & 0 & $0.0 \%$ & - \\
\hline IUD & 0 & $0.0 \%$ & 0 & $0.0 \%$ & - \\
\hline SGA & 1 & $2.5 \%$ & 0 & $0.0 \%$ & \\
\hline Total & 40 & $100 \%$ & 40 & $100 \%$ & \\
\hline
\end{tabular}

Amongst cases there was only one baby $(2.5 \%)$ who was small for gestation age and kept in nursery for observation for 4 hours. On the other hand in control group $39(97.5 \%)$ baby were alive and healthy, $1(2.5 \%)$ baby had meconium aspiration syndrome for which baby was kept in nursery for observation. There was no perinatal mortality observed amongst control group. Also, in both cases and controls there were no intrauterine death and still birth.

It was observed that there were $13(32.5 \%)$ NICU admissions amongst cases and 4 (10.0\%) amongst controls. Hence, there was a significant correlation between NICU stay amongst patient with history of first trimester surgical abortion ( $\mathrm{p}$ value 0.027 ). Most common cause for admission was fetal distress, for which baby was observed in nursery for max period of 24 hours.

\section{DISCUSSION}

Aim of study was to study association between Induced surgical abortion and fetal- maternal outcomes (low birth weight baby, NICU stay, small for gestation age, fetal complications, preterm birth, and mode of delivery) in subsequent pregnancy. Following are the Effect of first trimester surgical abortion on maternal outcomes in ongoing pregnancy:

\section{Maternal outcome}

In present study $9(22.5 \%)$ of babies born were late preterm (born at 32 to 37 week) in control and 21 $(52.5 \%)$ amongst cases with previous history of surgical abortion. The results for preterm birth was significant amongst cases ( $\mathrm{p}$ value: 0.006 ). There was 3.8 times risk of late preterm birth in cases as compared to control. There was one $(2.5 \%)$ baby born amongst cases who was very preterm (born less than 28 weeks of gestation) and none in the control group. It was observed that there were 16 caesarean (40\%) amongst cases and 15\% amongst control. Indication for cesarean section in most of cases was either fetal distress, placenta Previa.

Also, the rate of cesarean section were comparable in the study conducted by Dhaliwal in Chandigarh where 58 $(19.3 \%)$ cesarean amongst the cases and $47(15.7 \%)$ in control group. Cesarean rate was significantly higher among cases compared to controls. ${ }^{6}$

The maternal outcomes were compared with many studies, results were supported by Moreau $\mathrm{C}$ et al, Weijin Zhou MD, Martius JA et.al, Dhaliwal et al and it showed similar result. ${ }^{5-8}$

\section{Fetal outcome}

This study concluded that $47.5 \%$ (odds ratio: 6.8 ) of baby born to mothers taken as cases were low birth weight $(1.5$ to $2.5 \mathrm{~kg}$ ) and $5(12.5 \%)$ babies were born weighing the same among controls. Thus showing significant correlation between baby weight (ranging from 1.5 to 2.5 $\mathrm{kg}$ ) among cases $(<0.001)$. However there was only 1 baby $(2.5 \%)$ amongst cases who was extremely low birth weight, weighing $800 \mathrm{gms}$ at birth. There was one $(2.5 \%)$ live birth each in cases and controls each There was no significant association between very low birth weight and history of first trimester abortion (odds ratio: 1.79) amongst cases.

The results of our study were consistent with study conducted by Zhou W, Sorensen and Olsen J, Annapurna P et al. ${ }^{6,9}$

In the present study conducted it was seen that there was no significant correlation between neonatal outcome in cases compared to controls ( $\mathrm{p}$ value: 1.000). Out of 40 deliveries 37 .

(92.5\%) of babies in cases and $97.5 \%$ of babies amongst control were alive and healthy. One baby (less than 
$800 \mathrm{gms}$ ), accounting for $2.5 \%$ of total cases died immediately after birth. There was no mortality seen in control group. One baby $(2.5 \%)$ amongst cases was small for gestation age and none amongst controls. In both the groups there were no intrauterine death and no still births. Comparable results were seen by study conducted by Reime et al, Bhattacharya et al. ${ }^{10,4}$

There was no significant correlation seen between low Apgar score and history of first trimester surgical abortion. Most of the babies born in both cases and controls had Apgar score more than 7 at one minute of birth i.e. $82.5 \%$ amongst cases and $92.5 \%$ amongst controls. Similar observation was made by Klemetti et al in $2012 .^{11}$

A statistically significant correlation was seen between previous abortion and NICU stay. Not much studies could be obtain on the nicu stay however one such study was there conducted by Michel Makhlouf et al showed similar results. ${ }^{12}$

\section{CONCLUSION}

Surgical intervention for termination leads to damage to endometrium lining. This damage result in improper implantation of placenta in next pregnancy. This may lead to inadequate blood flow to baby, therefore resulting into fetal growth restriction.

Fetal growth restriction is associated with long term consequences such as: mental retardation due to intrauterine hypoxia, higher risk of cerebral palsy, delayed neurodevelopment causing delayed achievement of milestones. FGR and pre term labor is an important cause for prolonged NICU stay. Damage to endometrium and infection after surgical abortion is a risk factor for first trimester abortion in next pregnancy. Risk of infections, following surgical intervention are one of the causes of future fertility issues and higher risk of ectopic pregnancy after abortion. Cervical trauma during surgical abortion procedure can result into incompetency of cervical os, thus causing mid trimester abortion and increase risk of preterm labor. The risk of early premature births (EPBs) (<32 weeks gestation) and extremely early premature births (XPBs) $(<28$ weeks gestation) increases with increase in number of abortions.

Large studies have reported that a doubling of extremely premature birth risk after two prior surgical Induced abortions. Hence patient with induced abortion are at increased maternal and neonatal morbidity, therefore strict surveillance and monitoring is required in subsequent pregnancy.
Funding: No funding sources

Conflict of interest: None declared

Ethical approval: The study was approved by the Institutional Ethics Committee

\section{REFERENCES}

1. Beck C, Berry NS, Choijil S. Health system reform and safe abortion: A case study of Mongolia, Global Public Health. International Journal for Research, Policy and Practice. 2013;8:174-86.

2. Johnston, Robert WM. Indian abortion and live birth, 1971-2008 working paper. 2017. Available at: http://www.johnstonarchive.net/policy/abortion/india /ab-indias.html. Accessed on 04 ${ }^{\text {th }}$ May 2020.

3. Bhattacharya S. Reproductive outcomes following induced abortion: a national register based cohort study in Scotland: BMJ Open. 2012;2(4):e000911.

4. Johnson LG, Mueller BA, Daling JR. The relationship of placenta praevia and history of induced abortion.Int $\mathrm{J}$ Gynaecol Obstet. 2003;1(2):191-8.

5. Dhaliwal L K, Gupta K R, Gopalan S. Induced Abortion and subsequent pregnancy outcome. J Fam Welf. 2003;49:50-5.

6. Zhou W, Sorensen HT, Olsen J. Induced abortion and low birth weight in subsequent pregnancy. Int $\mathbf{J}$ Epidemiol. 2000;29(1):100-6.

7. Martius JA, Steck T, Oehler MK, Wulf KH. Risk factors associated with preterm (37 weeks) and early preterm birth ( 32 weeks): univariate and multivariate analysis of 106,345 singleton births from the 1994 statewide perinatal survey of Bavaria. Eur J Obstet Gynecol Reprod Biol. 1998;80:183-9.

8. Moreau C, Kaminski M, Ancel PY, Bouyer J, Escande B, Thiriez $G$ et al. Previous induced abortions and the risk of very preterm delivery: results of the EPIPAGE study. BJOG. 2005;112:4307.

9. Annapurna P, Devi T B, Devi B K. Pregnancy outcome following abortion. J Evid Based Med. 2015;2(41):6992-7002.

10. Reime B, Schücking BA, Wenzlaff P. Reproductive outcomes in adolescents who had a previous birth or an induced abortion compared to adolescents' first pregnancies. BMC Pregnancy Childbirth. 2008;8:4.

11. Klemetti R, Gissler M, Niinimäki M, Hemminki E. Birth outcomes after induced abortion:a nationwide register-based study of first births in Finland:Hum Reprod. 2012;27(11):331-520.

12. Makhlouf MA. Adverse pregnancy outcomes among women with prior spontaneous or induced abortions: Am J Perinatol. 2014;31(9):765-72.

Cite this article as: Tanwar K, Sarkar B, Chauhan A. To study the Feto-maternal outcome in cases with previous surgical intervention for first trimester abortion on subsequent pregnancy and to compare with cases without history of previous abortion. Int $\mathbf{J}$ Reprod Contracept Obstet Gynecol 2020;9:4227-31. 